\title{
The Use of Technology in Collegiate Aviation Programs
}

\author{
Deak Arch \\ Ohio University \\ Mark Sherman \\ Central Missouri State University
}

\begin{abstract}
With introduction of Technically Advanced Aircraft (TAA) and advanced Global Positioning Systems (GPS), a blind survey was designed to measure the extent of technology utilized in collegiate aviation programs. University Aviation Association (UAA) member institutions completed an online Likert Scale survey focusing on the perception of technology utilization within each aviation program. The survey questioned respondents regarding technology support, aircraft cockpit design, classroom accessories, internet resources, training facilities, and other miscellaneous areas regarding technology. The study was designed to aid university administrators when planning future technology implementation.
\end{abstract}

\section{INTRODUCTION}

The University Aviation Association (UAA) is a nonprofit organization consisting of 115 institutions representing academic and aviation industry with over 800 members. The UAA has strengthened aviation education and training within collegiate setting through scholarships, research, and student support. The organization provides a strong liaison between collegiate aviation and industry and continues to be at the forefront of aviation education and training improvement. Currently, there are 46 academic UAA member institutions providing aviation training and education.

UAA member institutions were selected for study participation due to their driving force within the aviation industry, leadership within the collegiate setting, and access to current technological advancements. This study was created to examine technology utilized within UAA member aviation programs. Respondents completed an online Likert Scale survey focused on their perception of technology used within UAA aviation programs. The focus of this study was to illustrate current technology trends and aid university administrators in future technology planning and implementation.

\section{REVIEW OF LITERATURE}

Past studies focused on effects of technology implementation upon transfer of learning. For example, Witiw and KellyBenjamin (1997) compared student performance in a technology-enhanced aviation meteorology course. They found the control group showed increased conceptual basic meteorology knowledge over two experimental groups. Howell, Denning, and Fizpatrick (2003) examined associated effects on university student achievement when provided with traditional printed lecture handouts versus electronically retrieved handouts. They found no statistical difference between electronic and traditional delivery of specific course materials.

Articles have focused on technology within classroom settings. Karp (2000) remarked on how computer-based training (CBT) and personal computer-based aviation training devices (PCATDs) aid in knowledge retention of aviation students. Burgener (2005) reported how technology implementation transfers digital video technology to overhead projectors in classroom settings. In "The Technological Revolution Comes to the Classroom,” Konza \& Johnston (1991) wrote, "faculty members need to see how creative and effective teachers change the curriculum, their assignments, the arrangement of the classroom, and the ways students interact when they introduce technology into their courses" (p.10).

The internet has become an integral part within the higher education system. However, this technology has not been without limitations. Simmons (2005) addressed concerns utilizing internet-based resources for use during instruction of undergraduate airline management 
courses. These concerns included information that was out-dated, unreliable, or not always available for retrieval.

With the emergence of technically advanced aircraft (TAA), technology has influenced the cockpit, as well as the classroom. This paper examined technology trends in UAA collegiate aviation programs.

\section{TECHNOLOGY SURVEY}

Forty-six UAA member institutions were invited by e-mail to participate in an online blind study consisting of twenty-two questions (See Appendix). The survey focused on different uses of technology within each institution's program. Seventy-three percent of UAA member institutions completed the online survey with seventy-nine percent of respondents being fouryear institutions.

Various programs offered in collegiate aviation were represented. Ninety-seven percent of the 34 respondents offered aviation flighttraining programs and eighty-five percent offered aviation management programs. Twentysix percent offered aviation maintenance (Airframe and/or Powerplant Maintenance) programs. (See Table 1).

Respondents ranked university support in utilization of technology on a scale ranging from poor to excellent. Over eighty percent of respondents indicated support from university administration, deans, and chairs ranging from average to excellent. Only one institution reported poor support from the chair of the department. (See Table 2)

Respondents ranked aircraft technology and indicated their choice from strongly agreeing, to strongly disagreeing with selected statements. Sixty-one percent agreed technically advanced aircraft were utilized in their programs. Eightyfive percent of institutions polled indicated use of Global Positioning Systems (GPS). (See Table 3)

Questions concerning technology use in the classroom were solicited. One hundred percent of respondents indicated they talked about aircraft technology in the classroom. Eighty-two percent indicated demonstrating aircraft technology with computer programs in the classroom. (See Table 4)

Participants were polled on technology use in classroom settings. Seventy-eight percent indicated computers were installed within the classroom. Ninety percent of respondents indicated installed VCRs, ninety-three percent indicated installed DVDs, and eighty-one percent indicated having LCD projectors within the classroom setting. (See Table 5)

Concerning software utilization, eighty-four percent indicated they were using some type of training program (Microsoft Flight Simulator ${ }^{\circledR}$, Elite ${ }^{\circledR}$, Jeppesen Sim Charts ${ }^{\circledR}$, etc.). Fifty-seven percent indicated use of technical training software such as Vector Aircraft Systems ${ }^{\circledR}$, aircraft system CBT training, etc. (See Table 6).

Next addressed were class management tools available to instructors. Ninety-three percent indicated use of PowerPoint ${ }^{\circledR}$, seventyfive percent indicated use of WebCT ${ }^{\circledR}$ or Blackboard $\AA$, and sixty-one percent conduct online quizzes. Fifty-eight percent conducted online assessments and sixty-five percent use online services to distribute class syllabi. Sixtyseven percent utilized WebCT ${ }^{\circledR}$ or Blackboard ${ }^{\circledR}$ to distribute documents used in the course including lecture outlines, handouts, and website links associated with classroom content. Fiftyeight percent used some sort of multimedia presentation (Avi, Quicktime ${ }^{\circledR}$, mpeg, etc.); however, less than fifteen percent used $16 \mathrm{~mm}$ films and filmstrips. (See Table 7)

Table 1. Training Programs

\begin{tabular}{|c|c|c|}
\hline Training Programs Offered & Percentage of Respondents & Number of Respondents \\
\hline Aviation Flight Training & $97.06 \%$ & 33 \\
\hline Aviation Management & $85.29 \%$ & 29 \\
\hline Aircraft Maintenance & $26.47 \%$ & 9 \\
\hline Other (Not Listed) & $20.59 \%$ & 7 \\
\hline Air Traffic Control & $17.65 \%$ & 6 \\
\hline Avionic Maintenance & $14.71 \%$ & 5 \\
\hline Human Factors/Safety & $8.82 \%$ & 3 \\
\hline
\end{tabular}


Table 2. Support Received in Utilization of Technology

\begin{tabular}{|c|c|c|c|c|c|}
\hline \multicolumn{7}{|c|}{ Support Received in Utilization of Technology } \\
\hline & Excellent & Good & Average & Fair & Poor \\
\hline University Administration & $34.38 \%$ & $31.25 \%$ & $18.75 \%$ & $15.63 \%$ & $0 \%$ \\
& $(11)$ & $(10)$ & $(6)$ & $(5)$ & \\
\hline Deans & $43.75 \%$ & $21.88 \%$ & $18.75 \%$ & $15.63 \%$ & $0 \%$ \\
& $(14)$ & $(7)$ & $(6)$ & $(5)$ & \\
\hline Chairs & $53.13 \%$ & $25.00 \%$ & $12.50 \%$ & $6.25 \%$ & $3.13 \%$ \\
& $(17)$ & $(8)$ & $(4)$ & $(2)$ & $(1)$ \\
\hline
\end{tabular}

(Number of respondents are indicated inside of the parenthesis.)

Table 3. How Technology is Involved

\begin{tabular}{|l|c|c|c|c|c|}
\hline \multicolumn{7}{|c|}{ How Technology is Involved - Aircraft } \\
\hline & Strongly Agree & Agree & Neutral & Disagree & Strongly Disagree \\
\hline Technologically Advanced & $29.41 \%$ & $32.35 \%$ & $20.59 \%$ & $11.76 \%$ & $5.88 \%$ \\
Aircraft Utilized & $(10)$ & $(11)$ & $(7)$ & $(4)$ & $(2)$ \\
\hline Performance Management & $12.90 \%$ & $12.90 \%$ & $38.71 \%$ & $22.58 \%$ & $12.90 \%$ \\
System & $(4)$ & $(4)$ & $(12)$ & $(7)$ & $(4)$ \\
\hline Flight Management Systems & $18.18 \%$ & $18.18 \%$ & $39.39 \%$ & $12.12 \%$ & $12.12 \%$ \\
& $(6)$ & $(6)$ & $(13)$ & $(4)$ & $(4)$ \\
\hline GPS / RNAV / Loran & $50.00 \%$ & $35.29 \%$ & $8.82 \%$ & $2.94 \%$ & $2.94 \%$ \\
& $(17)$ & $(12)$ & $(3)$ & $(1)$ & $(1)$ \\
\hline
\end{tabular}

(Number of respondents are indicated inside of the parenthesis.)

Table 4. Classroom Aircraft Technology

\begin{tabular}{|l|c|c|c|c|c|}
\hline \multicolumn{7}{|c|}{ Classroom Aircraft Technology } \\
\hline & Strongly Agree & Agree & Neutral & Disagree & Strongly Disagree \\
\hline Talk About Aircraft Technology & $\begin{array}{c}68.75 \% \\
(22)\end{array}$ & $\begin{array}{c}31.25 \% \\
(10)\end{array}$ & $0 \%$ & $0 \%$ & $0 \%$ \\
\hline $\begin{array}{l}\text { Demonstrate Aircraft Technology } \\
\text { With Computer Programs }\end{array}$ & $\begin{array}{c}38.24 \% \\
(13)\end{array}$ & $\begin{array}{c}44.12 \% \\
(15)\end{array}$ & $\begin{array}{c}17.65 \% \\
(6)\end{array}$ & $0 \%$ & $0 \%$ \\
\hline $\begin{array}{l}\text { Do Not Talk About Aircraft } \\
\text { Technology }\end{array}$ & $0 \%$ & $0 \%$ & $\begin{array}{c}6.67 \% \\
(2)\end{array}$ & $\begin{array}{c}26.67 \% \\
(8)\end{array}$ & $\begin{array}{c}66.67 \% \\
(20)\end{array}$ \\
\hline
\end{tabular}

(Number of respondents are indicated inside of the parenthesis.)

Table 5. PC Based Technology

\begin{tabular}{|c|c|c|c|c|c|}
\hline \multicolumn{6}{|c|}{ PC Based Technology } \\
\hline & Strongly Agree & Agree & Neutral & Disagree & Strongly Disagree \\
\hline Classroom Installed Computers & $\begin{array}{c}63.64 \% \\
(21) \\
\end{array}$ & $\begin{array}{l}15.15 \% \\
(5)\end{array}$ & $\begin{array}{c}12.12 \% \\
(4)\end{array}$ & $\begin{array}{c}9.09 \% \\
(3)\end{array}$ & $0 \%$ \\
\hline Classroom Installed VCR & $\begin{array}{l}75.00 \% \\
(24)\end{array}$ & $\begin{array}{l}15.63 \% \\
(5)\end{array}$ & $\begin{array}{l}6.25 \% \\
(2)\end{array}$ & $\begin{array}{c}3.13 \% \\
(1)\end{array}$ & $0 \%$ \\
\hline Classroom Installed DVD & $\begin{array}{c}75.76 \% \\
(25)\end{array}$ & $\begin{array}{l}18.18 \% \\
(6)\end{array}$ & $\begin{array}{c}3.03 \% \\
(1)\end{array}$ & $\begin{array}{l}3.03 \% \\
(1)\end{array}$ & $0 \%$ \\
\hline $\begin{array}{l}\text { Classroom Installed LCD Type } \\
\text { Projector }\end{array}$ & $\begin{array}{l}67.65 \% \\
(23)\end{array}$ & $\begin{array}{l}14.71 \% \\
(5)\end{array}$ & $\begin{array}{l}8.82 \% \\
(3)\end{array}$ & $\begin{array}{l}2.94 \% \\
(1)\end{array}$ & $\begin{array}{l}5.88 \% \\
(2)\end{array}$ \\
\hline Portable LCD Type Projector & $\begin{array}{c}46.88 \% \\
(15)\end{array}$ & $\begin{array}{l}18.75 \% \\
(6)\end{array}$ & $\begin{array}{l}15.63 \% \\
(5)\end{array}$ & $\begin{array}{l}6.25 \% \\
(2)\end{array}$ & $\begin{array}{c}12.50 \% \\
(4)\end{array}$ \\
\hline $\begin{array}{l}\text { Multimedia Projection Booth in } \\
\text { Classroom }\end{array}$ & $\begin{array}{l}30.30 \% \\
(10)\end{array}$ & $\begin{array}{l}24.24 \% \\
(8)\end{array}$ & $\begin{array}{l}18.18 \% \\
(6)\end{array}$ & $\begin{array}{l}9.09 \% \\
\text { (3) }\end{array}$ & $\begin{array}{l}18.18 \% \\
(6)\end{array}$ \\
\hline
\end{tabular}

(Number of respondents are indicated inside of the parenthesis.) 
Table 6. Software

\begin{tabular}{|l|c|c|c|c|c|}
\hline \multicolumn{7}{|c|}{ Software } \\
\hline Utilized With Simulated & Strongly Agree & Agree & Neutral & Disagree & Strongly Disagree \\
Training Programs & $61.76 \%$ & $23.53 \%$ & $14.71 \%$ & $0 \%$ & $0 \%$ \\
\hline Technical Systems Software & $(21)$ & $(8)$ & $(5)$ & & \\
Utilized & $42.42 \%$ & $15.15 \%$ & $24.24 \%$ & $15.15 \%$ & $3.03 \%$ \\
$(1)$ & $(5)$ & $(8)$ & $(5)$ & $(1)$ \\
\hline
\end{tabular}

(Number of respondents are indicated inside of the parenthesis.)

Table 7. Instructor Class Management Tools

\begin{tabular}{|c|c|c|c|c|c|}
\hline \multicolumn{6}{|c|}{ Instructor Class Management Tools } \\
\hline & Strongly Agree & Agree & Neutral & Disagree & Strongly Disagree \\
\hline PowerPoint Presentations & $\begin{array}{l}82.35 \% \\
(28)\end{array}$ & $\begin{array}{c}11.76 \% \\
(4)\end{array}$ & $\begin{array}{l}2.94 \% \\
(1)\end{array}$ & $\begin{array}{l}2.94 \% \\
(1)\end{array}$ & $0 \%$ \\
\hline Blackboard / WebCT Utilization & $\begin{array}{l}51.52 \% \\
(17)\end{array}$ & $\begin{array}{l}24.24 \% \\
(8)\end{array}$ & $\begin{array}{c}12.12 \% \\
(4)\end{array}$ & $\begin{array}{l}6.06 \% \\
(2)\end{array}$ & $\begin{array}{l}6.06 \% \\
(2)\end{array}$ \\
\hline $\begin{array}{l}\text { Blackboard / WebCT Utilization } \\
\text { for Online Quiz }\end{array}$ & $\begin{array}{l}41.18 \% \\
(14)\end{array}$ & $\begin{array}{l}20.59 \% \\
\text { (7) }\end{array}$ & $\begin{array}{l}20.59 \% \\
\text { (7) }\end{array}$ & $\begin{array}{l}11.76 \% \\
(4)\end{array}$ & $\begin{array}{l}5.88 \% \\
(2)\end{array}$ \\
\hline $\begin{array}{l}\text { Blackboard / WebCT Utilization } \\
\text { for Online Assessment }\end{array}$ & $\begin{array}{l}35.29 \% \\
(12)\end{array}$ & $\begin{array}{l}23.53 \% \\
(8)\end{array}$ & $\begin{array}{l}23.53 \% \\
(8)\end{array}$ & $\begin{array}{l}8.82 \% \\
(3)\end{array}$ & $\begin{array}{l}8.82 \% \\
\text { (3) }\end{array}$ \\
\hline $\begin{array}{l}\text { Blackboard / WebCT Utilization } \\
\text { of Syllabi }\end{array}$ & $\begin{array}{c}40.63 \% \\
(13)\end{array}$ & $\begin{array}{c}25.00 \% \\
(8)\end{array}$ & $\begin{array}{l}18.75 \% \\
(6)\end{array}$ & $\begin{array}{l}6.25 \% \\
(2)\end{array}$ & $\begin{array}{c}9.38 \% \\
(3)\end{array}$ \\
\hline $\begin{array}{l}\text { Blackboard / WebCT Utilization } \\
\text { of Course Documents }\end{array}$ & $\begin{array}{c}41.18 \% \\
(14)\end{array}$ & $\begin{array}{l}26.47 \% \\
(9)\end{array}$ & $\begin{array}{l}17.65 \% \\
(6)\end{array}$ & $\begin{array}{l}5.88 \% \\
(2)\end{array}$ & $\begin{array}{l}8.82 \% \\
(3)\end{array}$ \\
\hline $\begin{array}{l}\text { Faculty Issued Computers / } \\
\text { Laptops }\end{array}$ & $\begin{array}{l}58.82 \% \\
(20)\end{array}$ & $\begin{array}{l}14.71 \% \\
(5)\end{array}$ & $\begin{array}{c}14.71 \% \\
(5)\end{array}$ & $\begin{array}{l}8.82 \% \\
(3)\end{array}$ & $\begin{array}{l}2.94 \% \\
(1)\end{array}$ \\
\hline Dry Erase Boards in Classrooms & $\begin{array}{l}65.63 \% \\
(21)\end{array}$ & $\begin{array}{c}18.75 \% \\
(6)\end{array}$ & $0 \%$ & $\begin{array}{l}6.25 \% \\
(2)\end{array}$ & $\begin{array}{c}9.38 \% \\
(3)\end{array}$ \\
\hline Smart Boards in Classrooms & $\begin{array}{l}12.90 \% \\
(4)\end{array}$ & $\begin{array}{l}16.13 \% \\
(5)\end{array}$ & $\begin{array}{l}22.58 \% \\
(7)\end{array}$ & $\begin{array}{l}22.58 \% \\
(7)\end{array}$ & $\begin{array}{l}25.81 \% \\
(8)\end{array}$ \\
\hline Chalk Boards in Classrooms & $\begin{array}{l}20.59 \% \\
(7)\end{array}$ & $\begin{array}{l}20.59 \% \\
(7)\end{array}$ & $\begin{array}{l}23.53 \% \\
(8)\end{array}$ & $\begin{array}{l}17.65 \% \\
(6)\end{array}$ & $\begin{array}{l}17.65 \% \\
(6)\end{array}$ \\
\hline $\begin{array}{l}\text { Transparencies Utilized in } \\
\text { Classroom Presentations }\end{array}$ & $\begin{array}{l}26.47 \% \\
(9)\end{array}$ & $\begin{array}{c}38.24 \% \\
(13)\end{array}$ & $\begin{array}{l}14.71 \% \\
\text { (5) }\end{array}$ & $\begin{array}{c}11.76 \% \\
(4)\end{array}$ & $\begin{array}{l}8.82 \% \\
(3)\end{array}$ \\
\hline $\begin{array}{l}\text { Videos Utilized in Classroom } \\
\text { Instruction }\end{array}$ & $\begin{array}{l}64.71 \% \\
(22)\end{array}$ & $\begin{array}{c}29.41 \% \\
(10)\end{array}$ & $\begin{array}{l}5.88 \% \\
(2)\end{array}$ & $0 \%$ & $0 \%$ \\
\hline $\begin{array}{l}\text { DVDs Utilized in Classroom } \\
\text { Instruction }\end{array}$ & $\begin{array}{l}67.65 \% \\
(23)\end{array}$ & $\begin{array}{l}23.53 \% \\
(8)\end{array}$ & $\begin{array}{l}8.82 \% \\
\text { (3) }\end{array}$ & $0 \%$ & $0 \%$ \\
\hline $\begin{array}{l}16 \text { mm Films Utilized in } \\
\text { Classroom Instruction }\end{array}$ & $\begin{array}{l}6.06 \% \\
(2)\end{array}$ & $\begin{array}{l}3.03 \% \\
(1)\end{array}$ & $\begin{array}{l}15.15 \% \\
(5)\end{array}$ & $\begin{array}{l}36.36 \% \\
(12)\end{array}$ & $\begin{array}{l}39.39 \% \\
(13)\end{array}$ \\
\hline $\begin{array}{l}\text { Film Strips Utilized in } \\
\text { Classroom Instruction }\end{array}$ & $\begin{array}{l}8.82 \% \\
(3)\end{array}$ & $\begin{array}{l}5.88 \% \\
(2)\end{array}$ & $\begin{array}{c}11.76 \% \\
(4)\end{array}$ & $\begin{array}{c}32.35 \% \\
(11)\end{array}$ & $\begin{array}{c}41.18 \% \\
(14)\end{array}$ \\
\hline $\begin{array}{l}\text { Avi, Quicktime, mpeg, etc. } \\
\text { Utilized in Classroom } \\
\text { Instruction }\end{array}$ & $\begin{array}{c}29.41 \% \\
(10)\end{array}$ & $\begin{array}{c}29.41 \% \\
(10)\end{array}$ & $\begin{array}{c}23.53 \% \\
(8)\end{array}$ & $\begin{array}{c}5.88 \% \\
(2)\end{array}$ & $\begin{array}{c}11.76 \% \\
(4)\end{array}$ \\
\hline
\end{tabular}

(Number of respondents are indicated inside of the parenthesis.) 
Table 8. Student Issued and Mandated Utilization of Laptops

\begin{tabular}{|c|c|c|c|c|c|}
\hline \multicolumn{6}{|c|}{ Student Issued and Mandated Utilization of Laptops } \\
\hline & $\begin{array}{l}\text { Strongly } \\
\text { Agree }\end{array}$ & Agree & Neutral & Disagree & $\begin{array}{l}\text { Strongly } \\
\text { Disagree }\end{array}$ \\
\hline $\begin{array}{l}\text { Mandated Utilization in Classroom } \\
\text { Setting }\end{array}$ & $\begin{array}{l}15.15 \% \\
(5)\end{array}$ & $\begin{array}{c}15.15 \% \\
(5)\end{array}$ & $\begin{array}{l}9.09 \% \\
(3)\end{array}$ & $\begin{array}{l}24.24 \% \\
(8)\end{array}$ & $\begin{array}{c}36.36 \% \\
(12)\end{array}$ \\
\hline $\begin{array}{l}\text { Student Computer Integrated into } \\
\text { Instructor Work Station }\end{array}$ & $\begin{array}{c}3.13 \% \\
(1)\end{array}$ & $\begin{array}{c}12.50 \% \\
(4)\end{array}$ & $\begin{array}{l}21.88 \% \\
(7)\end{array}$ & $\begin{array}{l}15.63 \% \\
(5)\end{array}$ & $\begin{array}{c}46.88 \% \\
(15)\end{array}$ \\
\hline $\begin{array}{l}\text { CBT Programs Specifically for Host } \\
\text { Institution }\end{array}$ & $\begin{array}{l}12.12 \% \\
(4)\end{array}$ & $\begin{array}{l}24.24 \% \\
(8)\end{array}$ & $\begin{array}{l}21.21 \% \\
(7)\end{array}$ & $\begin{array}{l}15.15 \% \\
(5)\end{array}$ & $\begin{array}{l}27.27 \% \\
(9)\end{array}$ \\
\hline
\end{tabular}

(Number of respondents are indicated inside of the parenthesis.)

UAA institutions responded to questions regarding student issued laptops. Twenty-four percent indicated required mandatory use in the classroom setting. Thirty-three percent indicated student laptop integration with the instructor workstation. Thirty-six percent responded that computer based training programs were specifically licensed to the host institution. (See Table 8)

Questions were asked to measure utilization of PCATDs within the training program. Thirtyseven percent indicated use of PCATDs in accordance with approved Training Course Outline (TCO) for students to log time. Seventysix percent of the respondents indicated use of PCATDs for increasing skill proficiency. Twenty-two percent of respondents reported charging for PCATD use. (See Table 9)

Respondents answered questions regarding simulator and/or flight training device (FTD) usage. Seventy-eight percent used simulators and/or FTDs able to simulate both single and multi-engine operations. Thirty percent used type specific simulation equipment. Fifty-nine percent used generic visual display systems.
Fifty-five percent indicated use of sophisticated visual displays in their simulators and/or FTDs. Sixty-one percent used visual displays during student evaluation. Eighty-four percent used the simulator and/or FTD to log time creditable for the approved TCO. Fifty-eight percent used simulators and/or FTDs for student improvement without logging time in the training syllabus; however, forty-six percent charged students for use of simulators and/or PCATDs for these skill mastery opportunities. Sixty-four percent had GPS units installed in the simulator and/or FTD; while forty percent had technically advanced aircraft representations in the simulator and/or FTD. (See Table 10)

The survey questioned respondents about incorporation of an approved TCO. Eighty-four percent incorporated the TCO into the training program with only one institution indicating no TCO incorporated. Forty-two percent conducted 14 Code of Federal Regulations (CFR) Part 61 training at the institution. Thirty-six percent indicated that the student was able to choose between a 14 CFR Part 61 and a 14 CFR Part 141 training syllabus. (See Table 11)

Table 9. Personal computer Aviation Training Device

\begin{tabular}{|l|c|c|c|c|c|}
\hline \multicolumn{7}{|c|}{ Personal Computer Aviation Training Device (PCATD) } \\
\hline & Strongly Agree & Agree & Neutral & Disagree & Strongly Disagree \\
\hline Time Logged Toward Rating & $29.41 \%$ & $8.82 \%$ & $14.71 \%$ & $23.53 \%$ & $23.53 \%$ \\
(TSO Approved) & $(10)$ & $(3)$ & $(5)$ & $(8)$ & $(8)$ \\
\hline Unlogged Student Skill & $38.24 \%$ & $38.24 \%$ & $8.82 \%$ & $5.88 \%$ & $8.82 \%$ \\
Mastery Opportunities & $(13)$ & $(13)$ & $(3)$ & $(2)$ & $(3)$ \\
\hline Time on PCATD Charged & $11.76 \%$ & $11.76 \%$ & $20.59 \%$ & $8.82 \%$ & $47.06 \%$ \\
& $(4)$ & $(4)$ & $(7)$ & $(3)$ & $(16)$ \\
\hline Time on PCATD Uncharged & $35.29 \%$ & $26.47 \%$ & $14.71 \%$ & $11.76 \%$ & $11.76 \%$ \\
& $(12)$ & $(9)$ & $(5)$ & $(4)$ & $(4)$ \\
\hline
\end{tabular}

(Number of respondents are indicated inside of the parenthesis.) 
Table 10. Simulator/Flight Training Device

\begin{tabular}{|l|c|c|c|c|c|}
\hline \multicolumn{7}{|c|}{ Simulator / Flight Training Device (FTD) } \\
\hline & Strongly Agree & Agree & Neutral & Disagree & Strongly Disagree \\
\hline Generic Simulator / FTD Utilized & $52.94 \%$ & $26.47 \%$ & $8.82 \%$ & $5.88 \%$ & $5.88 \%$ \\
& $(18)$ & $(9)$ & $(3)$ & $(2)$ & $(2)$ \\
\hline Type Specific Simulator / FTD & $15.15 \%$ & $15.15 \%$ & $24.24 \%$ & $15.15 \%$ & $30.30 \%$ \\
Utilized & $(5)$ & $(5)$ & $(8)$ & $(5)$ & $(10)$ \\
\hline Generic Visual Displays in & $31.25 \%$ & $28.13 \%$ & $15.63 \%$ & $6.25 \%$ & $18.75 \%$ \\
Simulator / FTD & $(10)$ & $(9)$ & $(5)$ & $(2)$ & $(6)$ \\
\hline Sophisticated Visual Displays in & $38.24 \%$ & $17.65 \%$ & $11.76 \%$ & $14.71 \%$ & $17.65 \%$ \\
Simulator / FTD & $(13)$ & $(6)$ & $(4)$ & $(5)$ & $(6)$ \\
\hline Visual Displays Utilized During & $26.47 \%$ & $35.29 \%$ & $11.76 \%$ & $11.76 \%$ & $14.71 \%$ \\
Training Evaluation & $(9)$ & $(12)$ & $(4)$ & $(4)$ & $(5)$ \\
\hline Time Logged Toward Rating (TSO & $61.76 \%$ & $23.53 \%$ & $5.88 \%$ & $2.94 \%$ & $5.88 \%$ \\
Approved) & $(21)$ & $(8)$ & $(2)$ & $(1)$ & $(2)$ \\
\hline Unlogged Student Skill Mastery & $23.53 \%$ & $35.29 \%$ & $17.65 \%$ & $8.82 \%$ & $14.71 \%$ \\
Opportunities & $(8)$ & $(12)$ & $(6)$ & $(3)$ & $(5)$ \\
\hline Unlogged Student Skill Mastery & $20.59 \%$ & $26.47 \%$ & $17.65 \%$ & $5.88 \%$ & $29.41 \%$ \\
Opportunities - Charged for Time & $(7)$ & $(9)$ & $(6)$ & $(2)$ & $(10)$ \\
\hline Unlogged Student Skill Mastery & $11.76 \%$ & $5.88 \%$ & $23.53 \%$ & $29.41 \%$ & $29.41 \%$ \\
Opportunities - Not Charged & $(4)$ & $(2)$ & $(8)$ & $(10)$ & $(10)$ \\
\hline GPS Installed in Simulator / FTD & $44.12 \%$ & $20.59 \%$ & $8.82 \%$ & $8.82 \%$ & $17.65 \%$ \\
& $(15)$ & $(7)$ & $(3)$ & $(3)$ & $(6)$ \\
\hline Technically Advanced Aircraft & $17.65 \%$ & $23.53 \%$ & $20.59 \%$ & $17.65 \%$ & $20.59 \%$ \\
Represented in Simulator / FTD & $(6)$ & $(8)$ & $(7)$ & $(6)$ & $(7)$ \\
\hline
\end{tabular}

(Number of respondents are indicated inside of the parenthesis.)

Table 11. Training Course Outline

\begin{tabular}{|c|c|c|c|c|c|}
\hline \multicolumn{6}{|c|}{ Training Course Outline (TCO) } \\
\hline & Strongly Agree & Agree & Neutral & Disagree & Strongly Disagree \\
\hline Incorporated into Training Program & $\begin{array}{c}63.64 \% \\
(21)\end{array}$ & $\begin{array}{l}21.21 \% \\
(7)\end{array}$ & $\begin{array}{l}12.12 \% \\
(4)\end{array}$ & $0 \%$ & $\begin{array}{l}3.03 \% \\
\text { (1) }\end{array}$ \\
\hline $\begin{array}{l}\text { Part } 61 \text { Training Utilized by } \\
\text { Institution }\end{array}$ & $\begin{array}{l}15.15 \% \\
(5)\end{array}$ & $\begin{array}{l}27.27 \% \\
(9)\end{array}$ & $\begin{array}{l}12.12 \% \\
\text { (4) }\end{array}$ & $\begin{array}{l}15.15 \% \\
(5)\end{array}$ & $\begin{array}{l}30.30 \% \\
(10)\end{array}$ \\
\hline $\begin{array}{l}\text { Student Choice of Part } 61 \text { / } 141 \\
\text { Training }\end{array}$ & $\begin{array}{l}15.15 \% \\
(5)\end{array}$ & $\begin{array}{l}21.21 \% \\
(7)\end{array}$ & $\begin{array}{l}9.09 \% \\
(3)\end{array}$ & $\begin{array}{l}18.18 \% \\
(6)\end{array}$ & $\begin{array}{l}36.36 \% \\
(12)\end{array}$ \\
\hline
\end{tabular}

(Number of respondents are indicated inside of the parenthesis.)

Federal Aviation Administration (FAA) written testing facilities were addressed within the survey. Sixty-eight percent indicated utilizing a Laser Grade testing facility. Twentyfour percent indicated using a Computerized Aviation Testing Service (CATS) testing facility. Only one institution indicated using an AVTEST testing facility. (See Table 12)

Questions were poised to determine commonality of aviation-related resources at UAA member institutions. Forty percent of respondents indicated having a remote library separate from the main university library, devoted to aviation resources. Fifty-seven percent attested that the aviation library consisted of generic aviation resource materials. Sixty-four percent expressed library contained material specific to aviation programs offered by the university. Among these holdings, ninety percent agreed with having aviation textbooks and reading materials available for students in addition to aviation periodicals. Ninety-three percent reported having aviation reference materials available in the library for student use. 
Eighty-one percent reported having multimedia holdings available for student use. (See Table 13)

Departmental computer assets and utilization were also included within the study. Ninety percent of polled institutions agreed with allowing students use of departmental computer assets. Seventy-two percent provided a standalone computer system for student use while ninety-six percent reported having internet capable student computers. Only one institution indicated not having an internet capable student computer available. Seventy-five percent expressed having FAA written test bank questions installed on departmental computers. Over eighty percent of respondents had word processing, PowerPoint ${ }^{\circledR}$, spreadsheet, and data base software installed on departmental computers; however, two institutions indicated not having the software installed. (See Table 14)

All respondents indicated using some sort of IBM or a derivative of an IBM computer. No departments indicated using Macintosh (Mac) computers or Linux operating systems. Although a Likert Scale was utilized in the answering of the questions for this section, the researchers agree that a "yes/no" format would be more accurate when performing any replication of this survey. (See Table 15)
Inquiries were made concerning use of preflight weather stations. Seventy-eight percent had a weather station with aviation weather products available for student use. Eighty-seven percent had a telephone available for use during a preflight briefing and ninety-six percent had a personal computer (PC) available for weather briefings prior to flight. Although a Likert Scale was utilized in the answering of the questions for this section, the researchers agree that a "yes/no" format would be more accurate when performing any replication of this survey. (See Table 16)

Weather reporting services were examined. Seventy-seven percent strongly agreed with having an Automated Surface Observing System (ASOS) or Automated Weather Observing System (AWOS) located on the airport where student training was performed while forty-nine percent indicated having some sort of National Oceanic and Atmospheric Association (NOAA) weather reporting facility. Although a Likert Scale was utilized in the answering of the questions for this section, the researchers agree that a "yes/no" format would be more accurate when performing any replication of this survey. (See Table 17)

Table 12. Federal Aviation Administration Testing Facility

\begin{tabular}{|l|c|c|c|c|c|}
\hline \multicolumn{7}{|c|}{ Federal Aviation Administration Testing Facility } \\
\hline & Strongly Agree & Agree & Neutral & Disagree & Strongly Disagree \\
\hline Lasergrade Testing Utilized & $59.38 \%$ & $9.38 \%$ & $9.38 \%$ & $6.25 \%$ & $15.63 \%$ \\
& $(19)$ & $(3)$ & $(3)$ & $(2)$ & $(5)$ \\
\hline CATS Testing Utilized & $21.88 \%$ & $3.13 \%$ & $21.88 \%$ & $6.25 \%$ & $46.88 \%$ \\
& $(7)$ & $(1)$ & $(7)$ & $(2)$ & $(15)$ \\
\hline AVTEST Testing Utilized & $3.33 \%$ & $0 \%$ & $26.67 \%$ & $13.33 \%$ & $56.67 \%$ \\
& $(1)$ & & $(8)$ & $(4)$ & $(17)$ \\
\hline Owned and Operated by the & $56.25 \%$ & $6.25 \%$ & $15.63 \%$ & $6.25 \%$ & $15.63 \%$ \\
Aviation Department & $(18)$ & $(2)$ & $(5)$ & $(2)$ & $(5)$ \\
\hline Owned and Operated by University & $15.15 \%$ & $3.03 \%$ & $12.12 \%$ & $18.18 \%$ & $51.52 \%$ \\
Testing Services & $(5)$ & $(1)$ & $(4)$ & $(6)$ & $(17)$ \\
\hline On Airport / University Property & $21.21 \%$ & $6.06 \%$ & $6.06 \%$ & $15.15 \%$ & $51.52 \%$ \\
but Owned by Outside Entity & $(7)$ & $(2)$ & $(2)$ & $(5)$ & $(17)$ \\
\hline
\end{tabular}

(Number of respondents are indicated inside of the parenthesis.) 
Table 13. Aviation Library

\begin{tabular}{|c|c|c|c|c|c|}
\hline \multicolumn{6}{|c|}{ Aviation Library } \\
\hline & Strongly Agree & Agree & Neutral & Disagree & Strongly Disagree \\
\hline $\begin{array}{l}\text { Remote Library Separate from Main } \\
\text { University Library Dedicated to } \\
\text { Aviation }\end{array}$ & $\begin{array}{c}29.41 \% \\
(10)\end{array}$ & $\begin{array}{l}11.76 \% \\
(4)\end{array}$ & $\begin{array}{l}20.59 \% \\
(7)\end{array}$ & $\begin{array}{l}17.65 \% \\
(6)\end{array}$ & $\begin{array}{c}20.59 \% \\
(7)\end{array}$ \\
\hline Generic to All Aviation & $\begin{array}{c}36.36 \% \\
(12)\end{array}$ & $\begin{array}{c}21.21 \% \\
(7)\end{array}$ & $\begin{array}{c}30.30 \% \\
(10)\end{array}$ & $\begin{array}{l}6.06 \% \\
(2)\end{array}$ & $\begin{array}{l}6.06 \% \\
(2)\end{array}$ \\
\hline $\begin{array}{l}\text { Program Specific Material (Flight, } \\
\text { Maintenance, Management, etc.) }\end{array}$ & $\begin{array}{l}35.29 \% \\
(12)\end{array}$ & $\begin{array}{l}29.41 \% \\
(10)\end{array}$ & $\begin{array}{l}26.47 \% \\
(9)\end{array}$ & $0 \%$ & $\begin{array}{l}8.82 \% \\
(3)\end{array}$ \\
\hline
\end{tabular}

(Number of respondents are indicated inside of the parenthesis)

Table 14. Departmental Computer Assets

\begin{tabular}{|c|c|c|c|c|c|}
\hline \multicolumn{6}{|c|}{ Departmental Computer Assets } \\
\hline & Strongly Agree & Agree & Neutral & Disagree & Strongly Disagree \\
\hline Available for Student Use & $\begin{array}{l}72.73 \% \\
(24)\end{array}$ & $\begin{array}{l}18.18 \% \\
(6)\end{array}$ & $0 \%$ & $\begin{array}{c}3.03 \% \\
(1)\end{array}$ & $\begin{array}{c}6.06 \% \\
(2)\end{array}$ \\
\hline Stand Alone Student Computer System & $\begin{array}{l}51.52 \% \\
(17)\end{array}$ & $\begin{array}{c}21.21 \% \\
(7)\end{array}$ & $\begin{array}{c}9.09 \% \\
(3)\end{array}$ & $\begin{array}{l}3.03 \% \\
(1)\end{array}$ & $\begin{array}{l}15.15 \% \\
(5)\end{array}$ \\
\hline Internet Capable Student Computers & $\begin{array}{l}84.85 \% \\
(28)\end{array}$ & $\begin{array}{l}12.12 \% \\
(4)\end{array}$ & $0 \%$ & $\begin{array}{l}3.03 \% \\
(1)\end{array}$ & $0 \%$ \\
\hline $\begin{array}{l}\text { FAA Written Test Bank Questions } \\
\text { Installed }\end{array}$ & $\begin{array}{l}58.82 \% \\
(20)\end{array}$ & $\begin{array}{l}17.65 \% \\
(6)\end{array}$ & $\begin{array}{c}11.76 \% \\
(4)\end{array}$ & $\begin{array}{l}5.88 \% \\
(2)\end{array}$ & $\begin{array}{l}5.88 \% \\
(2)\end{array}$ \\
\hline Word Processing Capable & $\begin{array}{l}82.35 \% \\
(28)\end{array}$ & $\begin{array}{c}8.82 \% \\
(3)\end{array}$ & $\begin{array}{l}2.94 \% \\
(1)\end{array}$ & $\begin{array}{l}2.94 \% \\
(1)\end{array}$ & $\begin{array}{l}2.94 \% \\
(1)\end{array}$ \\
\hline PowerPoint Capable & $\begin{array}{l}85.29 \% \\
(29)\end{array}$ & $\begin{array}{l}11.76 \% \\
(4)\end{array}$ & $0 \%$ & $\begin{array}{c}2.94 \% \\
\text { (1) }\end{array}$ & $0 \%$ \\
\hline Spreadsheet Capable & $\begin{array}{l}85.29 \% \\
(29) \\
\end{array}$ & $\begin{array}{c}11.76 \% \\
(4)\end{array}$ & $0 \%$ & $\begin{array}{l}2.94 \% \\
(1)\end{array}$ & $0 \%$ \\
\hline Database Capable & $\begin{array}{l}76.47 \% \\
(26)\end{array}$ & $\begin{array}{l}11.76 \% \\
(4)\end{array}$ & $\begin{array}{l}5.88 \% \\
(2)\end{array}$ & $\begin{array}{l}2.94 \% \\
(1)\end{array}$ & $\begin{array}{l}2.94 \% \\
(1)\end{array}$ \\
\hline
\end{tabular}

(Number of respondents are indicated inside of the parenthesis.)

Table 15. Type of Computers

\begin{tabular}{|l|c|c|c|c|c|}
\hline \multicolumn{7}{|c|}{ Type of Computers in Department } \\
\hline & Strongly Agree & Agree & Neutral & Disagree & Strongly Disagree \\
\hline Macintosh & $0 \%$ & $0 \%$ & $\begin{array}{c}13.79 \% \\
(4)\end{array}$ & $\begin{array}{c}10.34 \% \\
(3)\end{array}$ & $\begin{array}{c}75.86 \% \\
(22)\end{array}$ \\
\hline $\begin{array}{l}\text { IBM or IBM Equivalent (Dell, } \\
\text { Gateway, Hewlett Packard, etc.) }\end{array}$ & $\begin{array}{c}85.29 \% \\
(29)\end{array}$ & $\begin{array}{c}14.71 \% \\
(5)\end{array}$ & $0 \%$ & $0 \%$ & $0 \%$ \\
\hline Linux & $0 \%$ & $0 \%$ & $\begin{array}{c}10.34 \% \\
(3)\end{array}$ & $\begin{array}{c}20.69 \% \\
(6)\end{array}$ & $\begin{array}{c}68.97 \% \\
(20)\end{array}$ \\
\hline
\end{tabular}

(Number of respondents are indicated inside of the parenthesis.) 
Table 16. Aircraft Preflight Weather Station

\begin{tabular}{|c|c|c|c|c|c|}
\hline \multicolumn{6}{|c|}{ Aircraft Preflight Weather Station } \\
\hline & Strongly Agree & Agree & Neutral & Disagree & Strongly Disagree \\
\hline Generic Weather Station & $\begin{array}{l}12.12 \% \\
(4)\end{array}$ & $\begin{array}{l}12.12 \% \\
(4)\end{array}$ & $\begin{array}{l}18.18 \% \\
(6)\end{array}$ & $\begin{array}{l}15.15 \% \\
(5)\end{array}$ & $\begin{array}{c}42.42 \% \\
(14)\end{array}$ \\
\hline Aviation Specific Weather Station & $\begin{array}{c}72.73 \% \\
(24)\end{array}$ & $\begin{array}{l}6.06 \% \\
(2)\end{array}$ & $\begin{array}{c}15.15 \% \\
(5)\end{array}$ & $\begin{array}{l}3.03 \% \\
(1)\end{array}$ & $\begin{array}{c}3.03 \% \\
(1)\end{array}$ \\
\hline Phone Available for Preflight Briefing & $\begin{array}{l}81.82 \% \\
(27)\end{array}$ & $\begin{array}{l}6.06 \% \\
(2)\end{array}$ & $\begin{array}{l}9.09 \% \\
(3)\end{array}$ & $0 \%$ & $\begin{array}{l}3.03 \% \\
(1)\end{array}$ \\
\hline $\begin{array}{l}\text { PC Available for Checking Weather } \\
\text { (DUAT, Internet, etc.) }\end{array}$ & $\begin{array}{l}87.50 \% \\
\text { (28) }\end{array}$ & $\begin{array}{l}9.38 \% \\
(3)\end{array}$ & $\begin{array}{l}3.13 \% \\
(1)\end{array}$ & $0 \%$ & $0 \%$ \\
\hline
\end{tabular}

(Number of respondents are indicated inside of the parenthesis.)

Table 17. On-Site Weather Reporting

\begin{tabular}{|c|c|c|c|c|c|}
\hline \multicolumn{6}{|c|}{ On-Site Weather Reporting } \\
\hline & Strongly Agree & Agree & Neutral & Disagree & Strongly Disagree \\
\hline ASOS on Field & $\begin{array}{c}34.38 \% \\
(11)\end{array}$ & $\begin{array}{c}9.38 \% \\
(3)\end{array}$ & $\begin{array}{c}25.00 \% \\
(8)\end{array}$ & $\begin{array}{c}6.25 \% \\
(2)\end{array}$ & $\begin{array}{c}25.00 \% \\
(8)\end{array}$ \\
\hline AWOS on Field & $\begin{array}{c}43.75 \% \\
(14)\end{array}$ & $\begin{array}{c}9.38 \% \\
(3)\end{array}$ & $\begin{array}{c}9.38 \% \\
(3)\end{array}$ & $\begin{array}{l}6.25 \% \\
(2)\end{array}$ & $\begin{array}{c}31.25 \% \\
(10)\end{array}$ \\
\hline $\begin{array}{l}\text { NOAA Weather Reporting } \\
\text { Capabilities }\end{array}$ & $\begin{array}{l}37.50 \% \\
(12)\end{array}$ & $\begin{array}{c}12.50 \% \\
(4)\end{array}$ & $\begin{array}{l}18.75 \% \\
(6)\end{array}$ & $\begin{array}{c}9.38 \% \\
(3)\end{array}$ & $\begin{array}{l}21.88 \% \\
(7)\end{array}$ \\
\hline
\end{tabular}

(Number of respondents are indicated inside of the parenthesis.)

Table 18. Aircraft Scheduling

\begin{tabular}{|l|c|c|c|c|c|}
\hline \multicolumn{7}{|c|}{ Aircraft Scheduling } \\
\hline & Strongly Agree & Agree & Neutral & Disagree & Strongly Disagree \\
\hline Individuals Employed to Schedule / & $72.73 \%$ & $12.12 \%$ & $9.09 \%$ & $3.03 \%$ & $3.03 \%$ \\
Dispatch Aircraft & $(24)$ & $(4)$ & $(3)$ & $(1)$ & $(1)$ \\
\hline No Individual Employed to Schedule & $6.06 \%$ & $3.03 \%$ & $15.15 \%$ & $12.12 \%$ & $63.64 \%$ \\
/ Dispatch Aircraft & $(2)$ & $(1)$ & $(5)$ & $(4)$ & $(21)$ \\
\hline Aircraft Scheduled Using Paper Log & $26.47 \%$ & $14.71 \%$ & $11.76 \%$ & $\begin{array}{c}8.82 \% \\
(3)\end{array}$ & $\begin{array}{c}38.24 \% \\
(13)\end{array}$ \\
\hline Aircraft Scheduled Using & $(9)$ & $(5)$ & $(4)$ & $(3)$ & $12.12 \%$ \\
Computer(s) & $54.55 \%$ & $18.18 \%$ & $12.12 \%$ & $3.03 \%$ & $(4)$ \\
\hline Aircraft Schedule Available on & $(18)$ & $(6)$ & $(4)$ & $(1)$ & $\begin{array}{c}\text { (1) } \\
\text { Internet }\end{array}$ \\
\hline
\end{tabular}

(Number of respondents are indicated inside of the parenthesis.)

Respondents were questioned in regards to aircraft scheduling. Eighty-four percent indicated that a specific individual(s) was employed to schedule and/or dispatch training aircraft. Seventy-two percent used a computer to schedule training flights while only forty percent of the institutions utilized a paper log to schedule training aircraft. Fifty-one percent provided access to the training schedule via the internet. Although a Likert Scale was utilized in the answering of the questions for this section, the researchers agree that a "yes/no" format would be more accurate when performing any replication of this survey. (See Table 18)

Respondents were asked how they viewed the importance of technology within their training program. All respondents indicated that technology was important in the classroom as well as in the aircraft. The majority of respondents indicated that technology was important in support of the department; however, one institution replied it was of less 
importance. The majority of institutions stated technology used in student support was important. (See Table 19)

Respondents were asked questions concerning future technology purchases. Eightyone percent employed technology enhanced aids during student classroom instruction. Seventytwo percent utilized technology aids for the department. Sixty-two percent used technologyenhanced aids for student training. Twenty-eight percent utilized technically advanced aircraft in their programs. While forty-six percent indicated plans to purchase technically advanced aircraft, only forty-four percent used upgraded avionics. Thirty-eight percent planned to purchase upgraded avionics. Seventy-two percent used GPS or RNAV systems within their respective program. (See Table 20)

\section{CONCLUSIONS}

With over 800 members, the UAA has established itself as a nationally recognized leader among collegiate aviation institutions. The analyzed data in this study reflected UAA member institutions use of current technology.

Modern computer access was common within the classroom settings. Over seventy-five percent of UAA institutions utilized advanced computer technology including PowerPoint ${ }^{\circledR}$ Presentations, classroom installed computers, aircraft programs, simulated training software, and use of Blackboard ${ }^{\circledR}$ or WebCT ${ }^{\circledR}$. In addition, computers were used by over half of the institutions to schedule aircraft and allow the schedule to be viewed on the internet. All UAA institutions polled indicated aircraft technology discussions within the classroom setting.

One of the most important statistics within this survey focused on use of technically advanced aircraft. Sixty one percent of the UAA institutions polled utilized technically advanced aircraft during the training of the students. These aircraft are equipped with the most modern avionics available on the market today.

Data presented by this study suggests that UAA member institutions keep up with current technology. The majority of UAA respondents felt that technology is very important in the classroom, aircraft, and department/student support system. This study demonstrated that UAA member institutions continue that leadership today within the technological realm by remaining on the forefront of technology innovations.

Table 19. Importance of Technology

\begin{tabular}{|l|c|c|c|}
\hline \multicolumn{3}{|c|}{ Importance of Technology } \\
\hline & Very Important & Important & Of Less Importance \\
\hline Technology in the Classroom & $\begin{array}{c}88.24 \% \\
(30)\end{array}$ & $\begin{array}{c}11.76 \% \\
(4)\end{array}$ & $0 \%$ \\
\hline Technology in the Aircraft & $81.82 \%$ & $18.18 \%$ & $0 \%$ \\
& $(27)$ & $(6)$ & $2.94 \%$ \\
\hline Technology Used in Supporting the & $82.35 \%$ & $14.71 \%$ & $(1)$ \\
Department & $(28)$ & $(5)$ & $5.88 \%$ \\
\hline Technology Used in Student Support & $76.74 \%$ & $17.65 \%$ & $(2)$ \\
& $(26)$ & $(6)$ & \\
\hline
\end{tabular}

(Number of respondents are indicated inside of the parenthesis.) 
Table 20. Future Technology Purchases

\begin{tabular}{|l|c|c|c|}
\hline \multicolumn{4}{|c|}{ Future Technology Purchases } \\
\hline & Use & Plan to Buy & No Plans to Buy \\
\hline Technology Aids in Classrooms & $81.82 \%$ & $12.12 \%$ & $6.06 \%$ \\
& $(27)$ & $(4)$ & $(2)$ \\
\hline Technology Aids for Department & $72.73 \%$ & $21.21 \%$ & $6.06 \%$ \\
& $(24)$ & $(7)$ & $(2)$ \\
\hline Technology Aids for Students & $62.50 \%$ & $15.63 \%$ & $21.88 \%$ \\
& $(20)$ & $(5)$ & $(7)$ \\
\hline Technically Advanced Aircraft & $28.13 \%$ & $46.88 \%$ & $25.00 \%$ \\
& $(9)$ & $(15)$ & $(8)$ \\
\hline Upgraded Avionics & $44.12 \%$ & $38.24 \%$ & $17.65 \%$ \\
& $(15)$ & $(13)$ & $(6)$ \\
\hline GPS / RNAV Systems & $72.73 \%$ & $15.15 \%$ & $12.12 \%$ \\
& $(24)$ & $(5)$ & $(4)$ \\
\hline
\end{tabular}

(Number of respondents are indicated inside of the parenthesis.) 


\section{REFERENCES}

Burgener, M. A. (2005, April). An examination of current technologies and methods for incorporating digital video into the aviation classroom. Paper presented at the Oklahoma Aviation Education Symposium.

Howell, C. D., Denning T. V., \& Fitzpatrick W. B. (2003). Traditional versus electronic information delivery: the effect on student achievement. International Journal of Applied Aviation Studies, 3, $207-214$.

Karp, M. R. (2000). University aviation education: an integrated model. Collegiate Aviation Review, $18,1-10$.

Kozna, R. B., \& Johnston, J. (1991). The technological revolution comes to the classroom. Change, 23, $10-23$.

Simmons, J. L., (2005, April). Internet-based resources for teaching undergraduate airline management courses: examples and uses. Paper presented at the Oklahoma Aviation Education Symposium.

Witiw, M. R., \& Kelly-Benjamin, K. (1997). Student performance in a technology-enhanced aviation meteorology course. Collegiate Aviation Review, 15, 33 - 42. 


\section{APPENDIX}

\section{SURVEY}

For each of the statements below, please indicate the extent of your agreement or disagreement by placing an $\mathrm{X}$ in the appropriate Column regarding your departmental utilization with technology

\section{Institution Classification}

2 Year Program

4 Year Program

\section{Select the Training Programs Offered by Your Institution}

Aviation Management

Aviation Flight Training

Avionic Maintenance

Aircraft Maintenance (Airframe and/or Powerplant)

Air Traffic Control

Human Factors / Safety

Other

\section{Support You Receive in Utilization of Technology}

University Administration

Deans

Chair

\author{
Excellent \\ Good \\ Average \\ Fair \\ Poor
}

Strongly Agree

Agree

Neutral

Disagree

Strongly Disagree

Strongly Agree

Agree

Neutral

Disagree

Strongly Disagree

Strongly Agree

Agree

Neutral

Disagree

Strongly Disagree

Multimedia Projection Booth in Classroom

\section{Software}

Utilized With Simulated Training Programs (Microsoft Flightsim, Elite, Sim Charts, Etc.)

Technical Systems Software (Vector Aircraft Systems, Aircraft Systems CBT Training, Etc.)
Strongly Agree

Agree

Neutral

Disagree

Strongly Disagree 


\section{Instructor Class Management Tools}

PowerPoint Presentations

Blackboard/Webct Utilization

Blackboard/Webct Utilization for Online Quiz

Blackboard/WebTV Utilization for Online Assessment

Blackboard/WebTV Utilization of Syllabi

Blackboard/WebTV Utilization of Course Documents

(Lecture Outlines, Handouts, Course Links, etc.)

Faculty Issued Computers/Laptops

Dry Erase Boards in Classroom

Smart Boards in Classroom

Chalk Boards in Classroom

Transparencies Utilized in Classroom Presentations

Videos Utilized in Classroom Instruction

DVD Utilized in Classroom Instruction

16mm Films Utilized in Classroom Instruction

Film Strips Utilized in Classroom Instruction

Avi, Quicktime, mpeg, etc. Presentations Utilized in

Classroom Instruction

\section{Student Issued and Mandated Utilization of Laptops}

Mandatory Utilization in Classroom Settings (Notes,

PowerPoints, Exam, Etc.)

Student Computer Integrated into Instructor Work Station

Computer Based Training Programs Specifically for Host Institution

10. PCATD

Time Logged Per FAR Toward Rating (TCO Syllabus Approved)

Unlogged Student Skill Mastery Opportunities

Time on PCATD Charged

Time on PCATD Uncharged

\section{Simulator / FTD}

Generic Simulator/FTD Utilized (Capable of Simulating

Multiple Aircraft Either Single or Multi Engine)

Type Specific Simulator/FTD Utilized

Generic Visual Displays in Simulator/FTD

Sophisticated Visual Displays in Simulator/FTD

Visual Displays Utilized During Training Evaluation

Time Logged per FAR Toward Rating (TCO Syllabus

Approved)

Opportunity for Unlogged Student Skill Mastery

Opportunity

Unlogged Skill Mastery Opportunity Charged for Time

Utilized

Unlogged Skill Mastery Opportunity Not Charged for

Time Utilized

GPS Installed in Simulator/FTD

Technically Advanced Aircraft Representation in

Simulator/FTD

Strongly Agree

Agree

Neutral

Disagree

Strongly Disagree

Strongly Agree

Agree

Neutral

Disagree

Strongly Disagree

Strongly Agree

Agree

Neutral

Disagree

Strongly Disagree

Strongly Agree

Agree

Neutral

Disagree

Strongly Disagree 


\section{TCO}

Incorporated into Training Program

Part 61 Training Utilized by Institution

Student Choice Part 141/61 Training Syllabus

\section{FAA Testing Facility}

Lasergrade Testing Utilized

Cats Testing Utilized

Avtest Testing Utilized

Owned and Operated by Aviation Department

Owned and Operated by University Testing Services

On Airport / University Property but Owned by Outside Entity

\section{Aviation Library}

Remote Library Separate from Main University Library

Dedicated to Aviation

Generic to All Aviation

Program Specific Material (Flight, Maintenance,

Management, Etc.)

\section{Aviation Library Holdings}

Aviation Textbooks, Reading Books on Shelf

Aviation Periodicals on Shelf

Aviation Reference Material on Shelf

Multimedia Holdings (VHS, DVD, 16 MM Films, Etc)

\section{Departmental Computer Assets}

Available for Student Use

Stand Alone Student Computer System

Internet Capable Student Computers

FAA Written Test Bank Questions Installed

Word Processing Capable

PowerPoint Capable

Spreadsheet Capable

Database Capable

\section{Type of Computers in Department}

MAC

IBM or IBM Equivalent (Hewlett Packard, Compaq, Dell,

Etc)

Lunix

\section{Aircraft Preflight Weather Station}

Generic Weather Station

Aviation Specific Weather Station

Phone Available for Preflight Briefing

PC Available for Checking Weather (DUAT, Internet, Etc.)
Strongly Agree

Agree

Neutral

Disagree

Strongly Disagree

Strongly Agree

Agree

Neutral

Disagree

Strongly Disagree

Strongly Agree

Agree

Neutral

Disagree

Strongly Disagree

Strongly Agree

Agree

Neutral

Disagree

Strongly Disagree

Strongly Agree

Agree

Neutral

Disagree

Strongly Disagree

Strongly Agree

Agree

Neutral

Disagree

Strongly Disagree

Strongly Agree

Agree

Neutral

Disagree

Strongly Disagree 


\section{On Site Weather Reporting}

ASOS on Field

AWOS on Field

NOAA Weather Reporting Capabilities

Strongly Agree

Agree

Neutral

Disagree

Strongly Disagree

\section{Aircraft Scheduling}

Specific Individual(s) Employed to Schedule/Dispatch

Aircraft

No Specific Individual(s) Employed to Schedule/Dispatch

Aircraft

Aircraft Scheduled Using Paper Log

Aircraft Scheduled Using Computer(s)

Aircraft Schedule Availible on Internet

Strongly Agree

Agree

Neutral

Disagree

Strongly Disagree

21. Importance of Technology

Technology in the Classroom

Very Important

Technology in the Aircraft

Technology Used in Supporting the Department

Technology Used in Student Support

Important

Of Less Importance

\section{Future Technology Purchases}

Technology Aids in Classroom

Technology Aids for Department

Use

Technology Aids for Students

Plan To Buy

Technically Advanced Aircraft

No Plans To Buy

Upgraded Avionics

GPS / RNAV Systems 